\title{
NEUROPEPTIDE Y Y2 RECEPTOR ACTIVATION PREVENTS METHAMPHETAMINE-INDUCED ENDOTHELIAL ALTERATIONS
}

Fabiana Ventura ${ }^{1}$, Ricardo A. Leitão ${ }^{1,2,3}$, Vanessa Coelho-Santos ${ }^{1,2,3}$, Ana Paula Silva ${ }^{1,2,3}$

1) Laboratory of Pharmacology and Experimental Therapeutics, Faculty of Medicine, University of Coimbra, Portugal; 2) Coimbra Institute of Clinical and Biomedical Research (iCBR), Faculty of Medicine, University of Coimbra, Portugal; ; 3) CNC.IBILI, University of Coimbra, Portugal; E-mail: fabi.ventura4@gmail.com

\section{INTRODUCTION}

Methamphetamine (METH) is a highly addictive psychostimulant largely consumed worldwide that leads to neurological and psychiatric disturbances. Its neurotoxicity has been related with oxidative stress, and glutamatergic and dopaminergic systems dysfunction $(1,2)$. Recently, the impact of METH on blood-brain barrier (BBB) integrity was pointed as a crucial event on brain alterations $(3,4)$. Our group already showed that METH increases BBB permeability by decreasing the levels of tight junction proteins that control the paracellular transport(5). Nevertheless, there is still no effective therapy against the effects of METH. The neuropeptide $\mathrm{Y}$ (NPY) is a 36-amino acid peptide(6) widely expressed in the CNS and PNS (7). Y1R and Y2R are the most expressed NPY receptors within brain parenchyma and their activities have been widely associated with psychiatric disorders such as anxiety, depression and post-traumatic stress disorder(8). Y2R is involved in memory loss prevention(2). Moreover, NPY has antiepileptic effects $(9,10)$, prevents neuronal apoptosis(11) and is a protective agent against METH-induced neuronal and microglial cell death(12), as well as a promoter of dentate gyrus neurogenesis(13). Several observations highlight the importance of NPY as a potential target in psychiatric disorders treatment. Noteworthy, clinical trials are ongoing to test the effect of intranasal NPY administration on mood/anxiety in health male volunteers (NCT 00748956) and in posttraumatic stress disorder (NCT 01533519)(8). Despite the promising protective effects of this neuropeptide, nothing is known about the role of NPY system on BBB function.

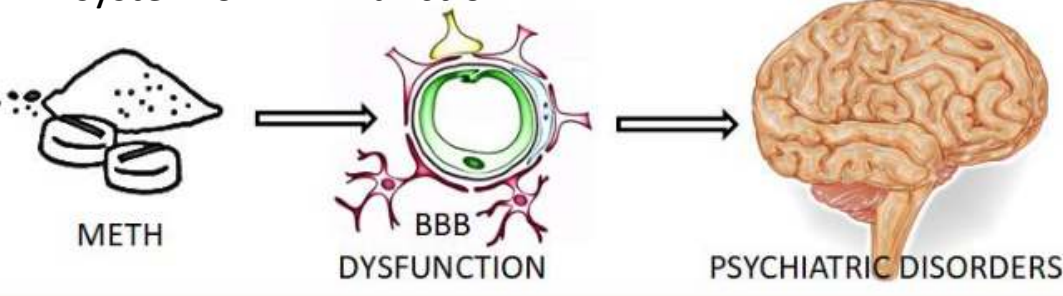

The aim was to uncover the role of NPY, and its receptors, on METH-induced endothelial cell dysfunction.

\section{MATERIAL AND METHODS}

Cell cultures: The human brain microvascular endothelial cell line (hCMEC/D3) was cultured in EBM-2 medium, supplemented with $1 \mathrm{ng} / \mathrm{mL}$ bFGF, lipid concentrate $(1: 100)$ $1.4 \mu \mathrm{M}$ hydrocortisone, $5 \mu \mathrm{g} / \mathrm{mL}$ acid ascorbic, $1 \%$ PenicillinStreptomycin, 5\% FBS, and $10 \mathrm{mM}$ HEPES. Medium was changed every 2 days until the cells reached confluence. hCMEC/D3 cells were seeded on culture plates coated with collagen type I and maintained at $37^{\circ} \mathrm{C}$ with $5 \% \mathrm{CO} 2$. Immunocytochemistry: hCMEC/D3 cells were left untreated (control) or incubated with METH $(100 \mu \mathrm{M})$ for $24 \mathrm{~h}$. Afterwards, cells were incubated with sheep anti-Y1R (1:200), rabbit anti-Y2R (1:100), overnight at $4^{\circ} \mathrm{C}$. After rinsed in $P B S$, cells were incubated with secondary antibodies (1:200), for $1 \mathrm{~h}$ at RT. Finally, cells were mounted with Dako fluorescent medium and images were captured using the LSM 710 Meta confocal microscope.

TUNEL Assay: In TUNEL assay hCMEC/D3 cells were incubated with $3 \mathrm{mM}$ METH for 24h. Pre-treatment with NPY

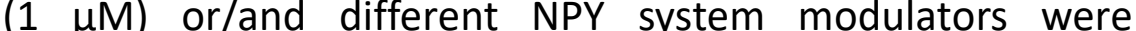
performed as described in the following timeline: Y1R agonist ([Leu31,Pro34]NPY) and antagonist (BIBP3226), Y2R agonist [NPY(13-36)] and antagonist (BIIE0246) $(1 \mu \mathrm{M}$ for all).

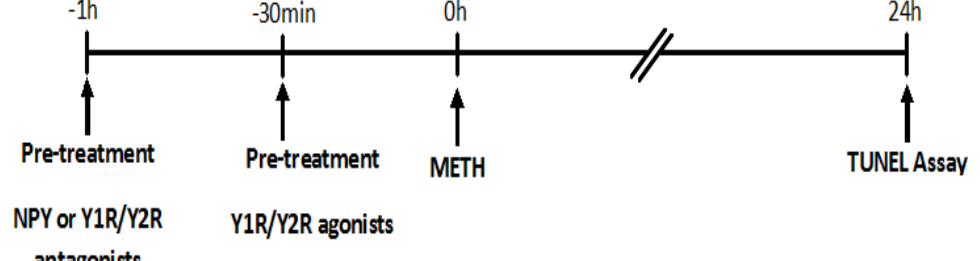

ROS Quantification: The hCMEC/D3 cells were seeded in black wells plates coated with collagen type I. Cells were treated with $3 \mathrm{mM}$ of METH and pre-treatment with NPY system agonists were performed 30 minutes before METH exposure. Together with appropriate treatments, cells were incubated with $\mathrm{H}_{2}$ DCFDA ( $5 \mu \mathrm{M}$ ) for 15 minutes or 30 minutes at $37^{\circ} \mathrm{C}$ in the dark. Fluorescence intensity was read (ex/em $485 / 528 \mathrm{~nm}$ ) and divided by the total amount of protein

\section{RESULTS}

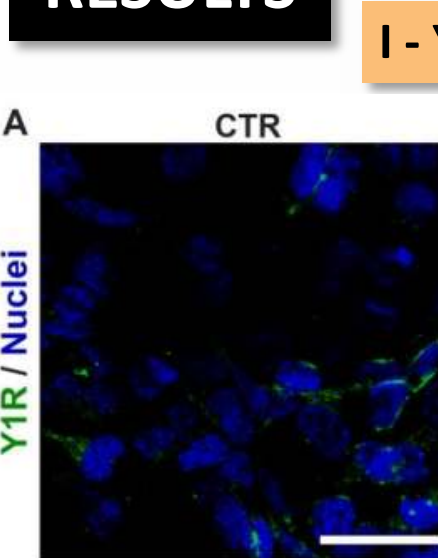

- Y1R and Y2R expression in human cerebral microvascular endothelial cells
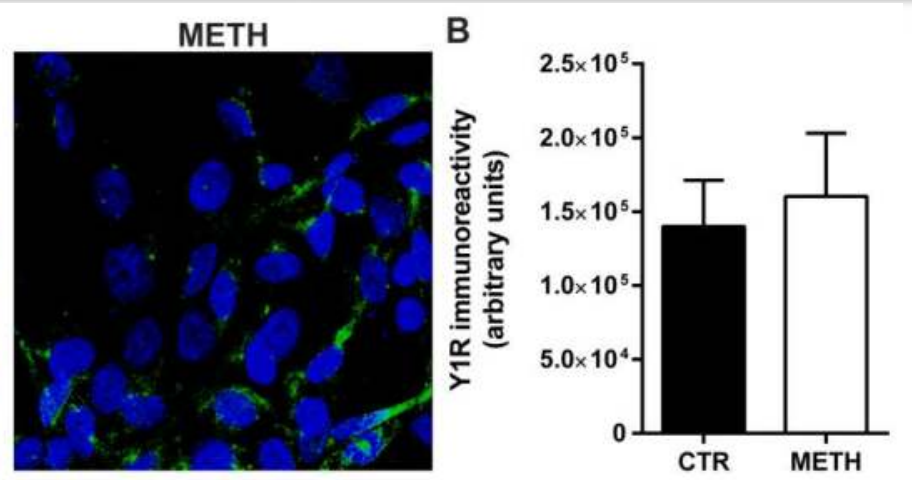

Figure 1 - Effect of METH on Y1R and Y2R expression in human brain endothelial cells. hCMEC/D3 cells were exposed to $3 \mathrm{mM}$ of METH for 24h. Representative immunocytochemistry fluorescence images for (A) Y1R (green), (C) Y2R (green), and Hoechst (blue, nuclei). Scale bar $=20 \mu \mathrm{m}$. First, (B) no alterations in Y1R
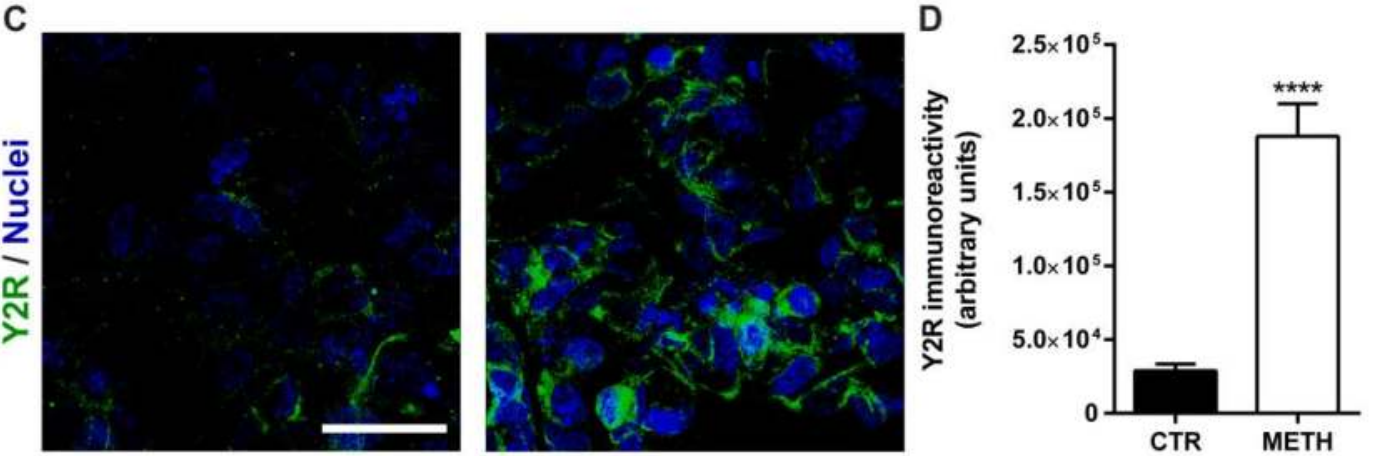
immunoreactivity was observed after $24 \mathrm{~h}$ of METH exposure. On the contrary, (D) significant increase in $Y 2 R$ immunoreactivity levels was observed in endothelial cells exposed to METH. Data are expressed as arbitrary units mean + S.E.M., $n=8, * * * * P<0.0001$ significantly different from control using Mann-Whitney test.

II - The activation of NPY2R prevents endothelial cell death induced by METH

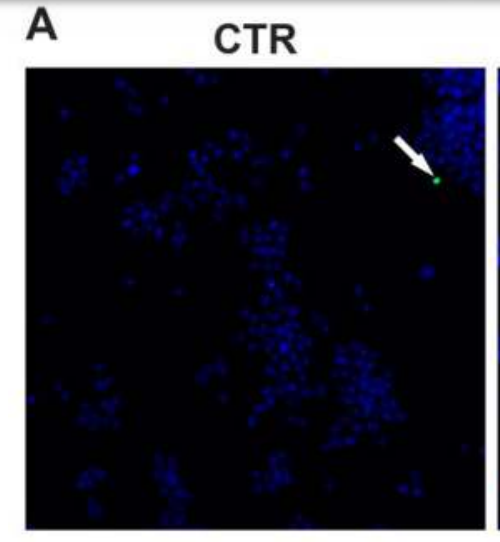

METH + NPY
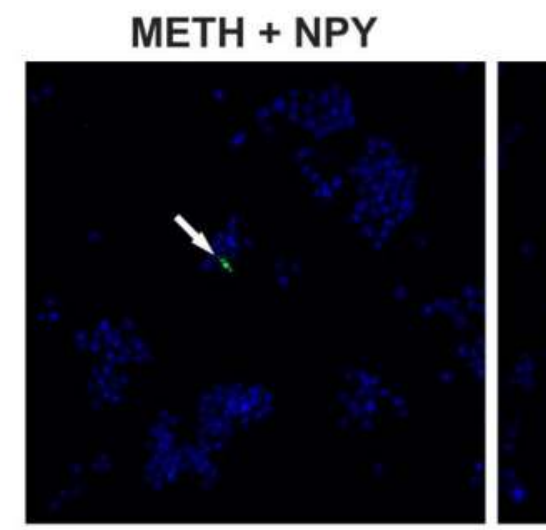

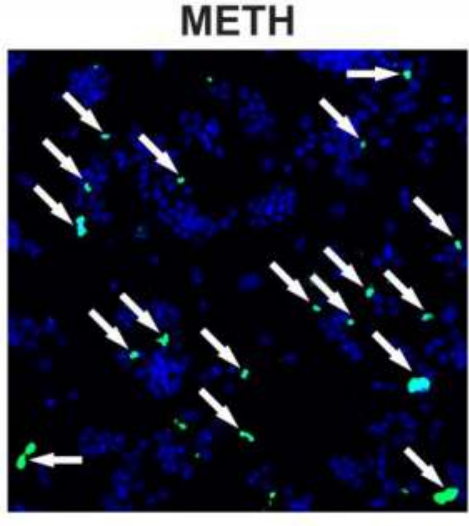

METH + 13-36

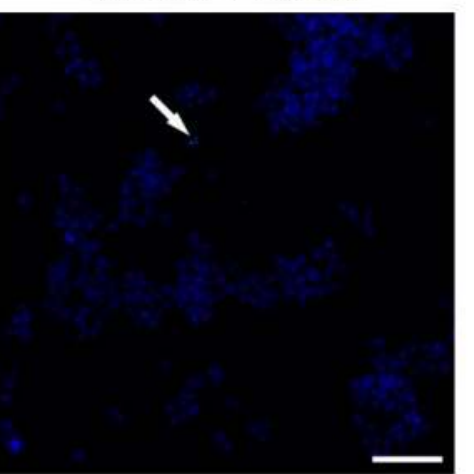

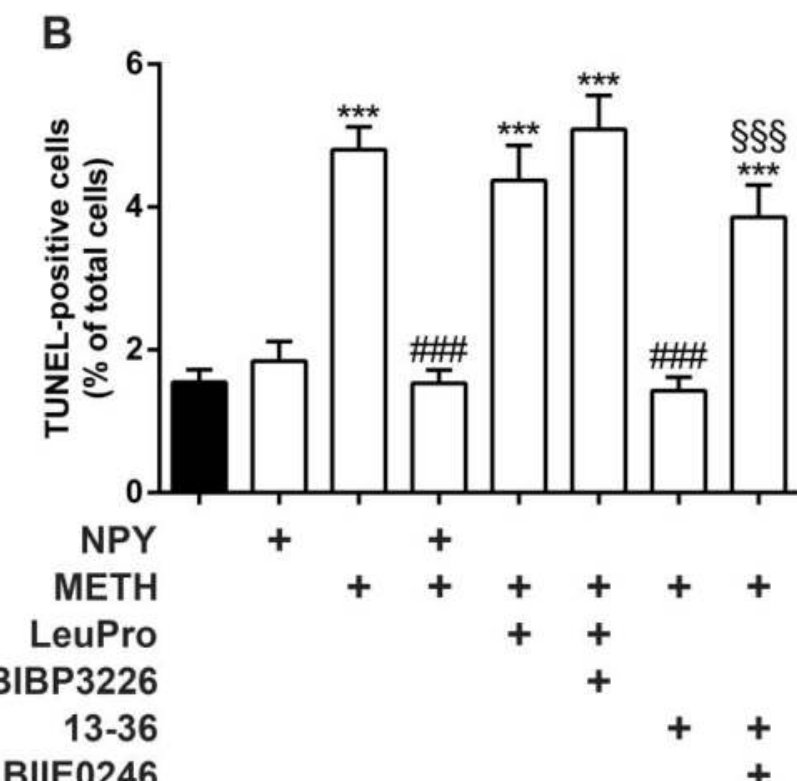

Fig.2 - Y2R activation prevents METH-induced endothelial cell death. hCMEC/D3 cells were exposed to METH (3mM for 24h) alone or with NPY $(1 \mu \mathrm{M})$, Y1R agonist (LeuPro; $1 \mu \mathrm{M}$ ) or Y2R agonist (13-36; $1 \mu \mathrm{M})$. Additionally, Y1R antagonist (BIBP3226) or Y2R antagonist (BIIE0246) were also used to block the effect of the specific agonists. (A) Representative images of TUNEL-positive cells (green; pointed by the white arrows) and nuclei (Hoechst; blue). Scale bar=40 $\mu \mathrm{m}$. (B) We observed a significant increase in cell death after exposure to METH. Interestingly, NPY prevented the cell death induced by METH. Moreover, only the activation of Y2R was able to inhibited the METH-induced cell death, and its selective effect was further proved using the $Y 2 R$ antagonist. The results are expressed as percentage of total cells. ${ }^{* * *} \mathrm{P}<0.001$, significantly different from control; \#\#\#<0.001, statistical significant when compared to $3 \mathrm{mM} M E T H ; \S \S \S \mathrm{P}<0.001$, significantly different from METH+13-36, using one-Way ANOVA followed by Bonferroni's post-test.

III - The activation of Y2R prevents METH inflammation via oxidative stress

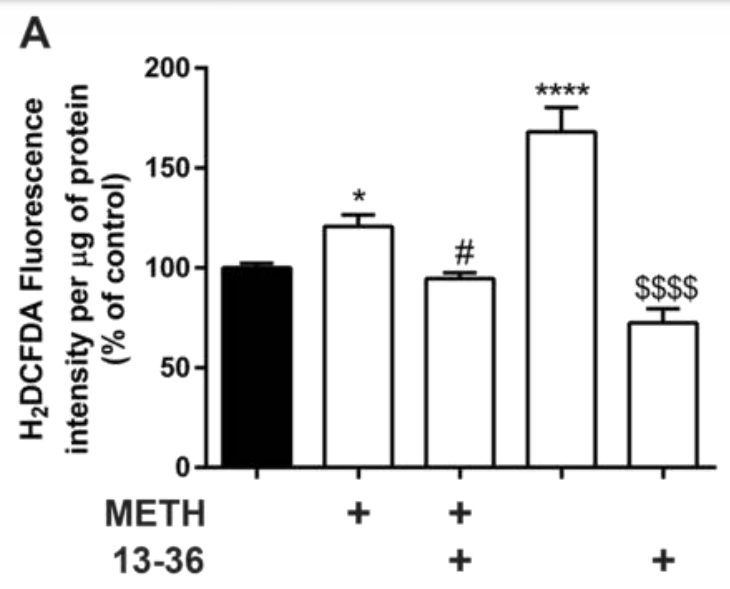

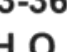

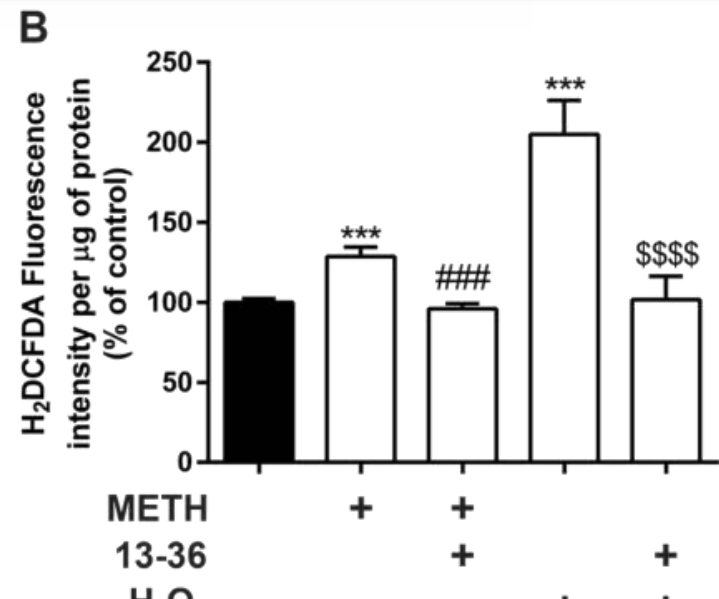

$\mathrm{H}^{2} \mathrm{O}_{2}$

Fig 3 - Y2R activation prevents METH-induced ROS production. $\mathrm{hCMEC} / \mathrm{D} 3$ cells were treated with $\mathrm{METH}(3 \mathrm{mM})$ alone or with $\mathrm{Y} 2 \mathrm{R}$ agonist [1 $\mu \mathrm{M} N \mathrm{NP}(13-36)]$ for $15 \mathrm{~min}$ or $30 \mathrm{~min}$. We observed a significant increase in METH-induced ROS production after (A) $15 \mathrm{~min}$, which is greater after (B) 30min. Interestingly, Y2R activation prevented oxidative stress in both timepoints evaluated. Results are expressed as mean $\%$ of control+SEM, ${ }^{*} \mathrm{P}<0.05,{ }^{* * *} \mathrm{P}<0.001,{ }^{* * * * P}<0.0001$ significantly different compared to control; \#P<0.05, $\# \# \# \mathrm{P}<0.001$ significant different compared to METH condition; $\$ \$ \$ \mathrm{P}<0.0001$ compared to $\mathrm{H}_{2} \mathrm{O}_{2}$ condition; using Kruskal-Wallis-test.

\section{SUMMARY}

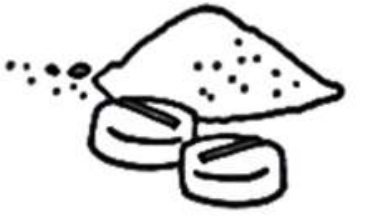

METH
- Human brain endothelial cells express both Y1R and Y2R.

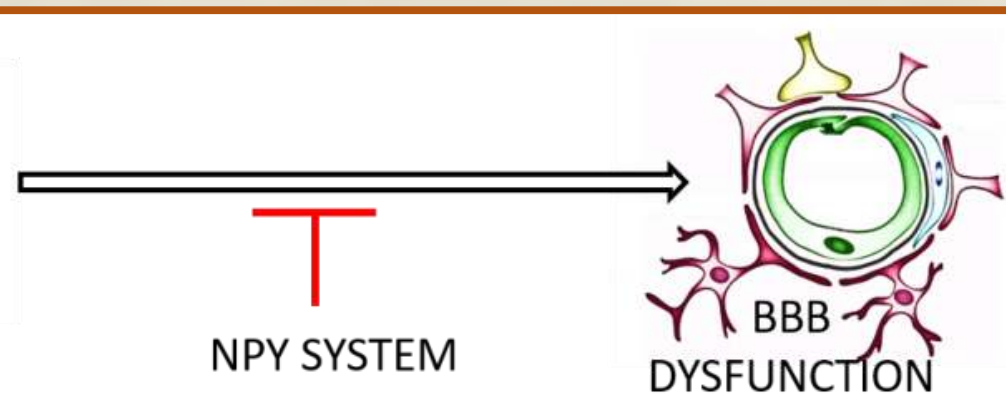

- NPY, by Y2R activation, has a protective effect against METH-induced cell death and oxidative stress.

- NPY can be a therapeutic target against BBB dysfunction and can be beneficial in psychiatric disorders. 